MVA combination not only proved safe, but also elicited large numbers of T cells and provided partial protection against a malaria challenge, extending to humans the promise that such vaccines have shown in monkeys and mice.

To get at just the right vaccine combination, the investigators evaluated 20 different conditions of priming and boosting by rolling volunteers from priming studies into boosting studies. The scope and breadth of this initial trial staked boundaries for future trials, conducting dose escalations and testing different numbers of immunizations. Under the most favorable conditions, single modality immunizations elicited fewer than 100 responding $\mathrm{T}$ cells per million white blood cells. In contrast, immunizations combining DNA priming with MVA boosting resulted in frequencies of responding cells that were ten times higher.

Impressively, T cells were elicited against all regions of the 557-amino acid malaria protein expressed in the immunogen. This contrasted with the low response against a 232-amino acid multiepitope string that had been fused to the $\mathrm{C}$ terminus of the expressed protein. The more robust response to the natural protein than the multiepitope string is good news. Despite the power of genetic engineering, expressing proteins is far easier than defining the histocompatibility types in a target population, identifying immunodominant epitopes for a pathogen and constructing a string of epitopes.

For those of us developing vaccines for which $\mathrm{T}$ cells, not antibody, will be the corre- late for protection, the study adroitly pioneers the screening of volunteers for vaccineelicited T cells. White blood cells were harvested and placed directly in enzyme-linked immunospot analyses to allow an ex vivo count of responding T cells. Assays were conducted on fresh, rather than frozen, cells to facilitate the scoring of proapoptotic T cells in peak effector responses. Consistent with the temporal pattern of T-cell responses, points were taken at one, rather than at two, weeks after boosting, when antibody responses would have been at their peak. The ability to detect enzyme-linked immunospot analysis responses was optimized by accepting, rather than attempting to zero, the nonspecific chatter of peptide stimulations. And finally, data were presented as geometric means as well as medians, to better represent patient-topatient variability in the numbers of elicited $\mathrm{T}$ cells.

This heterologous prime-boost protocol is but one of several currently in human trials. McMichael and Hanke, supported by the International AIDS Vaccine Initiative, have obtained promising results in DNA-MVA trials for HIV and AIDS, being conducted in England and Kenya ${ }^{8}$. In these trials, as in the current trial, the protein portion of the immunogen seems more effective than the multiepitope string at eliciting $\mathrm{T}$ cells. Approximately 800 volunteers are participating in trials for an AIDS vaccine developed by Merck that uses DNA priming and replication-defective adenovirus boosting and, more recently, replication-defective adenovirus priming followed by boosting with an avian poxvirus developed by Aventis. Many more modalities of priming and boosting are being tested in preclinical models. Provocatively, different protocols are raising different patterns of $\mathrm{CD}^{+}$and $\mathrm{CD}^{+}$T-cell responses ${ }^{9}$ and potentially different levels of $\mathrm{T}$ cells to dominant and subdominant epitopes. Thus, the rules of heterologous prime-boost immunizations are just beginning to be understood. Interesting science, as well as the potential to control major human pathogens, awaits those of us pursuing this powerful new approach to vaccination.

1. McConkey, S.J. et al. Enhanced T-cell immunogenicity in humans of plasmid DNA vaccines boosted by recombinant modified vaccinia virus Ankara. Nat. Med. 9, 729-735 (2003).

2. Schneider, J. et al. Induction of $\mathrm{CD}^{+} \mathrm{T}$ cells using heterologous prime-boost immunisation strategies. Immunol. Rev. 170, 29-38 (1999).

3. Amara, R.R. et al. Control of a mucosal challenge and prevention of AIDS by a multiprotein DNA/MVA vaccine. Science 292, 69-74 (2001).

4. Shiver, J.W. et al. Replication-incompetent adenoviral vaccine vector elicits effective anti-immunodeficiency virus immunity. Nature 415, 331-335 (2002).

5. Schneider, J. et al. Enhanced immunogenicity for $\mathrm{CD}^{+} \mathrm{T}$ cell induction and complete protective efficacy of malaria DNA vaccination by boosting with modified vaccinia virus Ankara. Nat. Med. 4, 397-402 (1998).

6. McShane, H., Brookes, R., Gilbert, S.C. \& Hill, A.V. Enhanced immunogenicity of CD4(+) T-cell responses and protective efficacy of a DNA-modified vaccinia virus Ankara prime-boost vaccination regimen for murine tuberculosis. Infect. Immun. 69, 681-686 (2001).

7. Irvine, K.R. et al. Enhancing efficacy of recombinant anticancer vaccines with prime/boost regimens that use two different vectors. J. Natl. Cancer Inst. 5, 1595-1601 (1997).

8. Hanke, T. et al. Development of a DNA-MVA/HIVA vaccine for Kenya. Vaccine 20, 1995-1998 (2002).

9. Robinson, H.L. New hope for an AIDS vaccine. Nat. Rev. Immunol. 2, 239-250 (2002).

\title{
Pausing paw proliferation
}

Methotrexate, perhaps the most widely prescribed drug for rheumatoid arthritis, slows the rapid proliferation of cells in the joints of patients with the disease. But its efficacy is limited by side effects that can include low sperm count and liver damage. In the May issue of the Journal of Immunology, Andreas Wunder et al. efficiently deliver methotrexate to the joints, increasing the dose where it is most needed. The investigators conjugated methotrexate to serum albumin, which enters fast-metabolizing cells such as those in inflamed joints. On the left is a mouse 5 minutes after injection with radiolabeled methotrexatealbumin conjugate, treated so that it has arthritic right paws. On the right is the same mouse after 13 hours, with accumulation of the drug in these paws. Mice treated with the conjugate fended off arthritis better than mice treated with methotrexate alone, and they accumulated less of the drug in their livers and kidneys. Similar uptake occurred in cells cultured from the joints of patients with rheumatoid arthritis. The combination, which has shown promise in human cancer trials, could minimize toxicity while boosting the effectiveness of methotrexate.

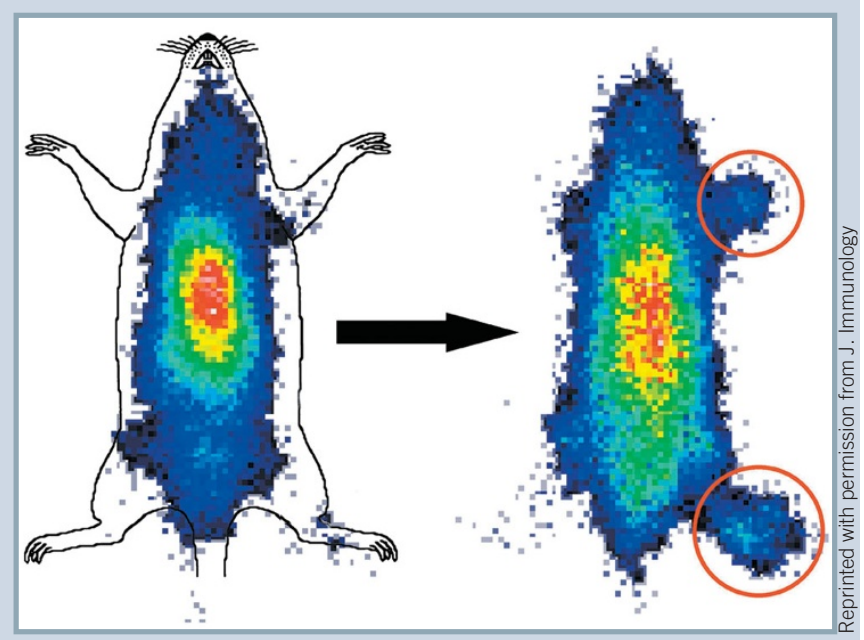

Charlotte Schubert 